\title{
Pattern formation driven by nematic ordering of assembling biopolymers
}

\author{
Falko Ziebert and Walter Zimmermann \\ Theoretische Physik, Universität des Saarlandes, D-66041 Saarbrücken, Germany
}

(Dated: November 13, 2018)

\begin{abstract}
The biopolymers actin and microtubules are often in an ongoing assembling/disassembling state far from thermal equilibrium. Above a critical density this leads to spatially periodic patterns, as shown by a scaling argument and in terms of a phenomenological continuum model, that meets also Onsager's statistical theory of the nematic-to-isotropic transition in the absence of reaction kinetics. This pattern forming process depends much on nonlinear effects and a common linear stability analysis of the isotropic distribution of the filaments is often misleading. The wave number of the pattern decreases with the assembling/disassembling rate and there is an uncommon discontinuous transition between the nematic and the periodic state.

PACS numbers: 47.54.+r, 64.70-p, 87.16.-b
\end{abstract}

Ongoing polymerization and depolymerization of actin and microtubule filaments are prominent examples for dissipative non-equilibrium phenomena in living cells [1], which are important for many different purposes, such as the cell motility and division or morphogenesis. Both substances show also an inherent propensity to pattern formation and active phenomena 2, 3, 4, 5, 6, 7, 8]. Like the famous example for rod-like particles, the Tobacco Mosaic Virus (TMV) 9], also actin and microtubule filaments may undergo with increasing density a well known transition to an orientational order [10, 11, 12], the socalled nematic order 13, 14]. By Onsager's seminal work 13, 14, 15, 16] this transition has been traced back to excluded volume interactions between the filaments. This statistical theory is valid for long filaments of fixed shape and infinite lifetime $\tau$, and it predicts near the transition also a phase separation into domains of isotropically oriented rods at low density and nematic domains of higher rod-density, which has also been observed for actin with an almost vanishing kinetics [11]. For a finite lifetime of actin and microtubule filaments, Onsager's statistical theory for the nematic order does not apply. Moreover, a finite $\tau$ limits the diffusive transport distance and the coarsening during the phase separation close to the orientational transition to a length scale of about $l_{D}=\sqrt{D_{\rho} \tau}$, with the filament diffusion constant $D_{\rho}$. According to our estimate we expect kinetically induced periodic patterns with a wavelength in the order of $10 \mu \mathrm{m}$. This is supported by current experiments 8$]$.

The effect of reaction kinetics on a phase separation has been investigated for a chemical and a biophysical example in Refs. [17, 18]. In both cases the transition to periodic patterns is supercritical and its onset as well as wavelength follows already from a linear stability analysis of the respective homogeneous basic state. Instead of these two competing states, near the orientational transition of filaments one has three different competing states, the spatially homogeneous isotropic state, the spatially homogeneous nematic one and the spatially inhomogeneous alternation between the isotropic and the nematic order. Here, the growth rate of perturbations of the basic state takes its maximum also at a finite wavenumber [19, 20], but this is not sufficient for a prediction of spatially inhomogeneous nonlinear states above the orientational transition. Instead of an inhomogeneous state, as suggested by the linear perturbation analysis, in the nonlinear regime one has an exchange of stability and the spatially homogeneous nematic state is often preferred, as described in this work. Therefore the bifurcation picture has to be explored by a nonlinear analysis, whereby an uncommon coarsening behavior at the nonlinear nematic-to-periodic transition has been found.

The generic scenario near the ordering transition is described in terms of a phenomenological model, which is introduced and analyzed at first without the reaction kinetics of the filaments. It is extended in the second part by the essential reaction steps as motivated by actin and microtubule polymerization.

Model without reaction kinetics. - In lyotropic liquid crystals the nematic order is forced beyond a critical rod density $\tilde{\rho}_{c}$ by excluded volume interactions [13] and the resulting local mean orientation of rod-like particles is described by the so-called director $\mathbf{n}(\mathbf{r})$ (with $\mathbf{n}=-\mathbf{n}$ ) 14]. Assuming rods of a single length and a uniform $\mathbf{n}$, then the largest eigenvalue $\tilde{\lambda}$ of the nematic order parameter tensor is sufficient for a description of the strength of the orientational order. $\tilde{\lambda}$ varies in the range $\left[\frac{1}{3}, 1\right]$, with $\tilde{\lambda}=\frac{1}{3}$ in the isotropic state and $\tilde{\lambda}=1$ for a uniform rod orientation [14, 21]. It is convenient to use the difference $\lambda:=\tilde{\lambda}-\frac{1}{3}$ and the dimensionless rod density $\rho \propto \tilde{\rho} V_{E}$, with $V_{E}=2 b L^{2}$ the excluded volume for rods of length $L$ and diameter $b$. For a constant orientation $\mathbf{n}$, as we assume in this work, spatial variations of $\rho$ and $\lambda$ also include spatially alternating isotropic and nematic ranges. Since for long rods the preferred director orientation is parallel to the isotropic-nematic interface [22], we assume spatial variations in the direction perpendicular to $\mathbf{n}$, which we call the $x$-direction. For this context we choose the phenomenological model for the conserved 
density $\rho(x, t)$ and for the unconserved field $\lambda(x, t)$

$$
\begin{aligned}
\partial_{t} \rho= & D_{\rho} \partial_{x}^{2}\left[-\lambda \rho-\delta_{\rho} \partial_{x}^{2} \rho+a_{\rho} \rho^{3}\right], \\
\partial_{t} \lambda= & -D_{r}\left[(1-\rho) \lambda-\frac{3}{2} \rho \lambda^{2}+\frac{9}{2} \rho \lambda^{3}\right] \\
& +D_{\lambda} \partial_{x}^{2}\left[(1-\rho) \lambda-\delta_{\lambda} \partial_{x}^{2} \lambda+a_{\lambda} \lambda^{3}\right] .
\end{aligned}
$$

In Eq. (1b), the part without spatial derivatives follows from the Smoluchowski equation for rigid rods by a moment approximation [21, 23] and the factor $\rho$ in front of the nonlinear terms reflects the excluded volume interaction. It determines also the homogeneous solutions

$$
\rho=\rho_{0}=\text { const. }, \quad \lambda_{0}=0, \quad \lambda_{ \pm}=\frac{1}{6} \pm \frac{1}{6} \sqrt{9-\frac{8}{\rho_{0}}} .
$$

$\lambda_{0}=0$ corresponds to the isotropic rod distribution that becomes linearly unstable with respect to nematic fluctuations beyond the critical density $\rho>\rho_{c}=1$, where they grow up to the homogeneously stable upper branch $\lambda_{+}$of the spatially uniform nematic order in Fig. 1b). Since the isotropic-nematic transition is of first order, both states coexist in a range $\frac{8}{9} \leq \rho \leq 1$.

Eq. (1a) is of the Cahn-Hilliard type 24]. Expressing its right hand side by a divergence of the current density $j_{\rho}(x)=-D_{\rho} \partial_{x} \mu(x)$ with $\mu(x)=-\lambda \rho-\delta_{\rho} \partial_{x}^{2} \rho+a_{\rho} \rho^{3}$, it takes the form of a conservation law for the rod-like particles. The first (nonlinear) term in Eq. (1a), i.e. $-D_{\rho} \partial_{x}^{2}(\lambda \rho)$, destabilizes the spatially homogeneous particle density for any finite value of $\lambda$ ( $\lambda$ is always positive) and mimics therefore Onsagers prediction [13, 16] that the free energy can be reduced, by separating the system into ranges of low rod density $\rho_{i}$ (isotropic) and high density $\rho_{a}$ (nematic). The second term describes an isotropic-nematic interface energy and the third term limits the modulation amplitudes of the density (see also below).

Theories involving the distribution function predict beyond the critical $\rho_{c}=1$ an instability of the isotropic distribution against inhomogeneous order parameter fluctuations [19, 23]. In Eq. (1b) this is taken into account by $\partial_{x}^{2}((1-\rho) \lambda)$ and the last two terms limit the wavenumber and the amplitude of the nonlinear modulations of $\lambda$. For intermediate values of $\rho$, Eqs. (1) have stationary kink solutions as shown in Fig. (1). The densities $\rho_{i}$ and $\rho_{a}$, in the isotropic and nematic range respectively, are determined by the two coefficients $a_{\rho}$ and $a_{\lambda}$, as described in the following. For stationary kinks as in Fig. 1:) the particle transport vanishes $j_{\rho}(x)=0$ and $\mu(x)=\mu_{i}=\mu_{a}$ is constant. Sufficiently far away from the kink $\rho_{a}$ and $\rho_{i}$ are constant too and one obtains the equation

$$
a_{\rho} \rho_{i}^{3}=-\lambda_{a} \rho_{a}+a_{\rho} \rho_{a}^{3},
$$

where $\lambda_{a}=\lambda\left(\rho_{a}\right)=\left(1+\sqrt{9-8 / \rho_{a}}\right) / 6$. The rotational term in Eq. 10 vanishes in the nematic range
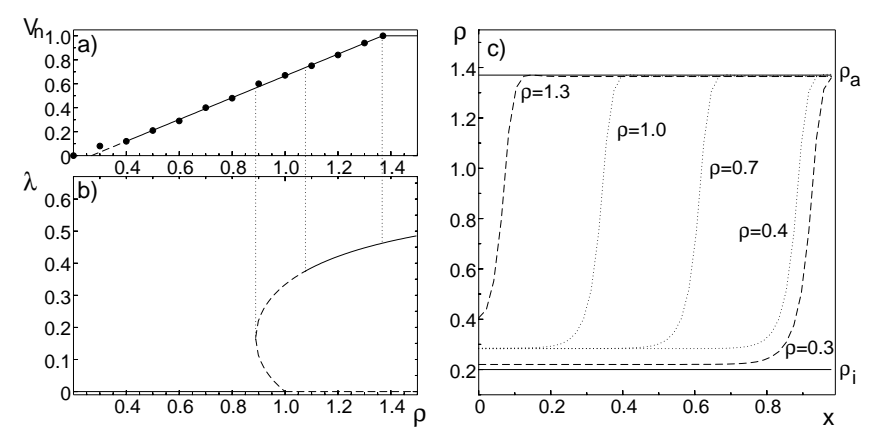

FIG. 1: Part b) shows the stable (solid) and the unstable branch (dashed) of the nematic order parameter $\lambda_{ \pm}$as a function of the rod density $\rho$, cf. Eq. (2). Part c) shows stable kink solutions of Eqs. (1) interpolating between the nematic $\left(\rho=\rho_{a}\right)$ and the isotropic range $\left(\rho=\rho_{i}\right)$. The nematic volume fraction $V_{n}$ as a function of $\rho$ is given in a). Parameters of the model: $D_{r}=0.1, D_{\rho}=D_{\lambda}=0.3, a_{\rho}=0.25, a_{\lambda}=$ $2.0, \delta_{\rho}=\delta_{\lambda}=0.1$. For this set, the whole system is in the homogeneous nematic state for $\rho_{0}>1.367$ (i.e $V_{n}=1$ ).

as well as trivially in the isotropic range. Defining $j_{\lambda}(x)=-D_{\lambda} \partial_{x} \nu(x)$, since in the isotropic range $\lambda_{i}$ is zero, $\nu_{i}$ is zero as well. To prevent a current through the interface, the total current, i.e. just $j_{\lambda}$ in the nematic region, has to vanish and it follows $\nu_{a}=\nu_{i}=0$ leading to

$$
\left(1-\rho_{a}\right) \lambda_{a}+a_{\lambda} \lambda_{a}^{3}=0 .
$$

As $\lambda_{a}$ is known, from this equation the anisotropic density $\rho_{a}$ follows as a function of $a_{\lambda}$ (or vice versa) and $\rho_{i}$ is given with $\rho_{a}$ via Eq. (3) as a function of $a_{\rho}$ (or vice versa). Therefore, the two densities $\rho_{i}$ and $\rho_{a}$ may also be considered as input parameters that are obtained from different approaches as for instance from Refs. 13, 25] or possibly from experiments. Since $\rho_{i}$ and $\rho_{a}$ do not depend on the system size $L$, the kink position changes with the mean density $\rho_{0}$ as shown in Fig. 1] where the nematic volume fraction $V_{n}$ is given in terms of $\rho_{0}$.

Linear stability analysis. - For $\rho>\rho_{c}$ a linear stability analysis of the homogeneous isotropic state, cf. $\lambda=0$ and $\rho=\rho_{0}$, with respect to small periodic perturbations $\lambda_{1}, \rho_{1} \propto \exp (\sigma t \pm i q x)$ gives the wave number dependence of the growth rate $\sigma(q)$ as shown for a set of parameters in Fig. 2a). This shape of $\sigma(q)$ with a positive value at $q=0$ and a maximum at a finite value of $q$ is typical for the unstable isotropic state and is in agreement with similar results for microscopic models [19]. A linear stability analysis of the homogeneous nematic state $\lambda_{ \pm}$in terms of microscopic models is rather involved 23]. For our phenomenological model, however, the determination of $\sigma(q)$ is a straightforward task and its typical shape at the unstable nematic branch is shown in Fig. 20). Along the dashed part of the curve in Fig. 10) the homogeneous nematic state is linear unstable. Between our result and the linear stability described in [19] there is a major 
difference. In both cases $\sigma(q)$ for the isotropic state takes its maximum at a finite value of $q$ and has positive values for any $\rho>\rho_{c}$. This is somewhat in contradiction to Onsager's statistical theory, where inhomogeneous states (via phase separation) are only energetically preferred for a rod density below a maximum value $\rho<\rho_{a}$. In our model nonlinear effects stabilize the uniform nematic state for $\rho>\rho_{a}$ and it is unstable only along the dashed line in Fig. 1b). Simulations of Eqs. (1) confirm that inhomogeneous solutions $\lambda(x)$ and $\rho(x)$ only occur for a mean density $\rho_{0}$ smaller than $\rho_{a}$.
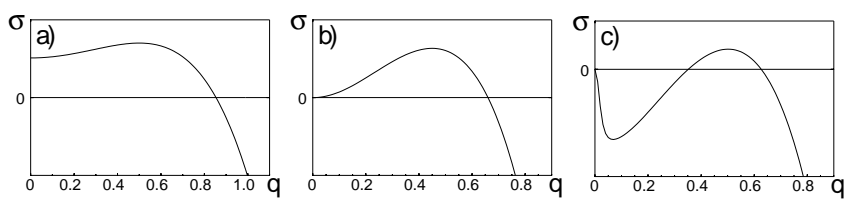

FIG. 2: In part a) $\sigma(q)$ is shown for periodic perturbations of the isotropic state with $\rho_{0}>\rho_{c}$ and in part b) of the nematic state without kinetics and at the unstable branch in Fig. 10). In part c) $\sigma(q)$ is given for perturbations of the nematic state in the case with reaction kinetics and at the unstable branch in Fig. 31). In part a) and b) the parameters are $\rho_{0}=1.05$ and $\Sigma=0$ and in c) $\Sigma=0.003$ and $s=0.01 \Sigma$. The other parameters are as in Fig. 1]

Reaction kinetics drives pattern. - In cells and in vitro actin and microtubule filaments are usually out of equilibrium and, due to an ongoing assembly/disassembly reaction, filaments have a finite lifetime $\tau$. This reaction kinetics leads to a stationary length distribution of the filaments [11] or even to oscillatory polymerization [2, 26, 27]. During the phase separation at the isotropic-to-nematic transition, filaments are transported, but only over a lifetime-dependent distance of about $l_{D}=\sqrt{D_{\rho} \tau}$. Since the lifetime of filaments is a constant, much more subunits are released in the nematic range with a high density $\rho_{a}$ than in the isotropic range with a low density $\rho_{i}$. However, due to a much larger diffusion constant, the subunits are redistributed quickly, leading to a nearly homogeneous subunit density $m(x)$. Thus the number of nucleated filaments per unit time, which depends on $m(x)$, is weakly varying too. By this qualitative reasoning one expects a steady net transport of subunits from the nematic to the isotropic range and in the opposite direction a transport of filaments, whereby the latter one is limited to distances of the order of $l_{D}$ or smaller. This length restriction causes, instead of a large scale phase separation, a spatially periodic pattern with a wavelength in the order of $l_{D}$.

Along this qualitative reasoning the complexity of the biochemical reaction steps, involved during the assembly/disassembly of actin or microtubules, is not crucial for this wavelength limitation. For instance, actin and microtubules are usually met with a polydisperse length distribution. Since the slowest kinetic step and the small
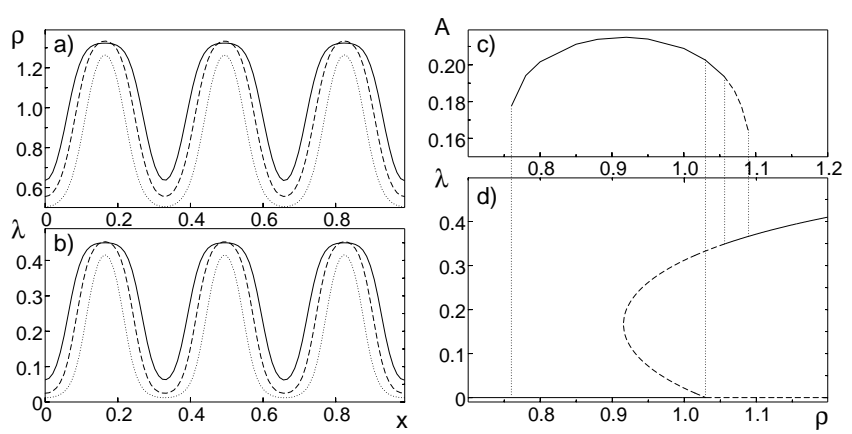

FIG. 3: Part a) and b) show periodic solutions $\rho(x)$ and $\lambda(x)$ of Eqs. (5) for $\rho_{0}=0.8$ (dotted), $\rho_{0}=0.95$ (dashed) and $\rho_{0}=1.056$ (solid), respectively. Part d) displays the stable homogeneous nematic branch (solid) and the unstable ones (dashed). In part c) the existence range of the stable (solid) and unstable (dashed) periodic patterns with the modulation amplitude $A$ of $\lambda(x)$ are given. Parameters are as in Fig. 1 with $D_{m}=10, \gamma=100, \Sigma=0.003, s=0.01 \Sigma$.

diffusion constant of the long filaments will govern the limitation, we discard the polydispersity and assume for the sake of simplicity that all filaments are of the same length. With a decay $\Sigma=\tau^{-1}$ and a nucleation rate $s$ of the filaments and a diffusion constant $D_{m}$ of the subunits one ends up finally with the three equations

$$
\begin{aligned}
\partial_{t} \rho & =D_{\rho} \partial_{x}^{2}\left[-\lambda \rho-\delta_{\rho} \partial_{x}^{2} \rho+a_{\rho} \rho^{3}\right]+s m-\Sigma \rho,(5 \mathrm{a}) \\
\partial_{t} \lambda= & -D_{r}\left[(1-\rho) \lambda-\frac{3}{2} \rho \lambda^{2}+\frac{9}{2} \rho \lambda^{3}\right] \\
& +D_{\lambda} \partial_{x}^{2}\left[(1-\rho) \lambda-\delta_{\lambda} \partial_{x}^{2} \lambda+a_{\lambda} \lambda^{3}\right]-\Sigma \lambda,(5 \mathrm{~b}) \\
\partial_{t} m= & D_{m} \partial_{x}^{2} m-\gamma s m+\gamma \Sigma \rho .
\end{aligned}
$$

The constant $\gamma$ is a measure for the number of subunits per rod-like particle. In the nematic range oriented filaments are lost, but new ones are nucleated everywhere with an arbitrary orientation which have to relax to the local mean orientation by rotational diffusion. Accordingly, there is only a decay term in Eq. (5b) which can be justified also microscopically 23]. This reaction kinetically caused partial loss of the orientational order leads to a higher critical density for the isotropic-to-nematic transition $\rho_{c}=1+\Sigma / D_{r}$. In the presence of reactive steps the rates $s$ and $\Sigma$ determine the mean rod density in terms of the monomer density $\rho=s \Sigma^{-1} m$. The spatially homogeneous solutions of Eqs.(5) are

$$
\rho=\rho_{0}, \quad \lambda_{0}=0, \quad \lambda_{ \pm}=\frac{1}{6} \pm \frac{1}{6} \sqrt{9-\frac{8}{\rho_{0}}\left(1+\frac{\Sigma}{D_{r}}\right)},
$$

where $\lambda_{ \pm}(\rho)$ are depicted as a function of the mean density $\rho$ in Fig. $3 \mathrm{~d}$ ). In a certain parameter range $\lambda_{+}$may become unstable with respect to periodic perturbations $\lambda_{1}, \rho_{1} \propto \exp (\sigma t \pm i q x)$ and the wave number dependence of the growth rate $\sigma(q)$ has a typical shape as in Fig. 2r). Compared to the case without kinetics as 
in Fig. 2b), long wavelength perturbations are now suppressed and only perturbations with a finite wave number grow. The parameter range of the unstable homogeneous $\lambda_{+}$branch is indicated by the dashed and the stable one by the solid line in Fig. $3 \mathrm{~d}$ ). The unstable range of the nematic branch decreases with increasing coefficients $\delta_{\rho}$ and $\delta_{\lambda}$ and with increasing and moderate values of the decay rate $\Sigma$. Periodic states occurring at the unstable nematic branch are shown in Fig. 37) and b) for three different mean densities $\rho_{0}$. The maxima of $\rho(x)$ come close to $\rho_{a}$, cf. Fig. 1] but the minima of $\rho(x)$ are considerably larger than $\rho_{i}$ in the case without kinetics. The solid line in Fig. 3r) indicates the range where the nonlinear periodic state is in coexistence with the homogeneous states. For $\rho>\rho^{*}$, along the dashed line in Fig. 3.), the periodic pattern becomes increasingly anharmonic, plateaus $\rho \sim \rho_{a}$ spread out and the valleys of low filament density in between become less and narrower by approaching the upper end of the dashed curve in Fig. 3r), a behavior that is rather uncommon 28]. At the left end of this curve the state remains periodic, the wavelength increases and the valleys spread out.

Conclusions.- A reaction kinetically driven pattern forming process is predicted near the isotropic-nematic (I-N) transition, which is supported by recent experiments on actin polymerization [8]. A phenomenological continuum model is introduced that reproduces the first order I-N transition in lyotropic liquid crystals, including the phase separation in its neighborhood and being in agreement with the statistical theory of Onsager. Periodic solutions arise due to a finite lifetime $\tau$ and a nucleation rate of the filaments. However, the correlation between a finite $\tau$ and the occurrence of periodic patterns is independent of the details of the model. Beyond a critical density $\rho_{c}$ the isotropic orientation of the filaments and below a certain $\rho^{*}$ the uniform nematic state becomes unstable against inhomogeneous perturbations. Hence for $\rho<\rho^{*}$ periodic states are favored and for our model the wavenumber varies as $q \propto \tau^{-0.17}$. However, $\rho_{c}$ increases and $\rho^{*}$ decreases with $\Sigma=\tau^{-1}$ and it may happen that $\rho^{*}<\rho_{c}$ holds, i.e. in a certain parameter range kinetics favors the uniform nematic state. Therefore, inhomogeneous states as predicted by a linear stability analysis of the isotropic state [19, 20] may be irrelevant due to nonlinear effects. This pattern formation process near the I-N transition is expected to be generic and may also apply to situations with different transport and filament accumulation mechanisms such as in the case of filament bundling [29, 30, 31]. Even though our description is very simplified and restricted to one spatial dimension, we expect that the basic physical mechanism also plays a crucial role for situations with polydisperse filament distributions [2, 11, 32], including living cells. Polydispersity favors periodic patterns and together with higher spatial dimensions this will give rise to an even larger variety of phenomena, to which forthcoming works are devoted.

We thank with great pleasure J. Dhont, B. Gentry, M. Hammele, J. Käs and K. Kawasaki for fruitful discussions.

[1] B. Alberts et al., Molecular Biology of the Cell (Garland Publishing, New York, 2001); H. Lodish et al., Molecular Cell Biology (W.H. Freeman, New York, 1999); J. Howard, Mechanics of Motor Proteins and the Cytoskeleton (Sinauer, Sunderland, 2001).

[2] M. F. Carlier et al., Proc. Natl. Acad. Sci. 84, 5257 (1987).

[3] E. Mandelkow et al., Science 246, 1291 (1989).

[4] J. Tabony and D. Job, Nature 346, 448 (1990).

[5] F. J. Nedelec, T. Surrey, A. C. Maggs, and S. Leibler, Nature 389, 305 (1997).

[6] O. Thoumine and A. Ott, J. Cell Sci. 110, 2109 (1997).

[7] D. Humphrey et al., Nature 416, 413 (2002).

[8] B. Gentry and J. Käs, (2003).

[9] F. C. Bawden, N. W. Pirie, J. D. Bernal, and I. Fankuchen, Nature 138, 1051 (1936).

[10] A. L. Hitt, A. R. Cross, and J. R. C. Williams, J. Bio. Chem. 265, 1639 (1990).

[11] J. Käs et al., Biophys. J. 70, 609 (1996).

[12] A. Suzuki, T. Maeda, and T. Ito, Biophys. J. 59, 25 (1991).

[13] L. Onsager, Ann. N.Y. Acad. Sci. 51, 627 (1949).

[14] P. G. de Gennes and J. Prost, The Physics of Liquid Crystals (Clarendon, Oxford, 1993).

[15] J. P. Straley, Mol. Cryst. \& Liq. Cryst. 22, 333 (1973).

[16] T. Odijk, Macromolecules 19, 2313 (1986).

[17] S. C. Glotzer, E. A. DiMarzio, and M. Muthukumar, Phys. Rev. Lett. 74, 2034 (1995).

[18] P. Fromherz and W. Zimmermann, Phys. Rev. E 51, R1659 (1995).

[19] T. Shimada, M. Doi, and K. Okano, J. Chem. Phys. 88, 7181 (1988).

[20] T. Liverpool and M. Marchetti, Phys. Rev. Lett. 90, $138102(2003)$.

[21] T. A. J. Lenstra, Z. Dogic, and J. K. G. Dhont, J. Chem. Phys. 114, 10151 (2001).

[22] A. J. Liu and G. H. Fredrickson, Macromolecules 29, 8000 (1996).

[23] F. Ziebert and W. Zimmermann, in preparation, 2003.

[24] J. W. Cahn and J. E. Hilliard, J. Chem. Phys. 28, 258 (1958).

[25] R. F. Kayser and H. J. Raveche, Phys. Rev. A 17, 2067 (1977).

[26] A. Marx and E. Mandelkow, Eur. Biophys. J. 22, 405 (1994).

[27] M. Hammele and W. Zimmermann, Phys. Rev. E 67, 021903 (2003).

[28] M. C. Cross and P. C. Hohenberg, Rev. Mod. Phys. 65, 851 (1993).

[29] K. Takiguchi, J. Biochem. 109, 502 (1991).

[30] K. Kruse, S. Camalet, and F. Jülicher, Phys. Rev. Lett. 87, 138101 (2001).

[31] A. Benedix, F. Ziebert, M. Hammele, and W. Zimmermann, unpublished, 2003.

[32] P. Sollich, J. Phys.: Cond. Mat. 14, R79 (2002). 